\title{
L'ordolibéralisme, unité et diversité
}

Ordoliberalism, unity and diversity

\section{Alain Alcouffe}

\section{(2) OpenEdition}

\section{Journals}

Édition électronique

URL : http://journals.openedition.org/ress/3954

DOI : $10.4000 /$ ress.3954

ISBN : 1663-4446

ISSN : 1663-4446

\section{Éditeur}

Librairie Droz

Édition imprimée

Date de publication : 15 décembre 2017

Pagination : 265-277

ISSN : 0048-8046

\section{Référence électronique}

Alain Alcouffe, "L'ordolibéralisme, unité et diversité », Revue européenne des sciences sociales [En ligne], 55-2 | 2017, mis en ligne le 15 décembre 2020, consulté le 19 janvier 2021. URL : http:// journals.openedition.org/ress/3954; DOI : https://doi.org/10.4000/ress.3954 


\title{
NOTES CRITIQUES
}

\section{L'ORDOLIBÉRALISME, UNITÉ ET DIVERSITÉ}

\author{
Alain Alcouffe (Université Toulouse-I Capitole)

\section{Patricia COMMUN, 2016, Les Ordolibéraux. Histoire d'un libéralisme à l'allemande Paris, Les Belles Lettres, 418 p.}

En un peu plus de 400 pages Patricia Commun nous offre une histoire de l'ordolibéralisme des années 1920 aux années 1960, un demi-siècle après la première présentation d'ensemble en français due à François Bilger (1964). Naturellement durant ce demi-siècle les études consacrées à l'ordolibéralisme en France n'ont pas manqué, à commencer par les travaux de Patricia Commun elle-même. Mais l'on peut citer aussi la thèse de Sylvain Broyer (2007), le livre de Jean Solchany (2015), les articles de Claire Mongouachon (20I5, 20I6), Raphaël Fèvre (20I5, 20I7a, 20I7b), une collection d'articles sous la direction d'Agnès Labrousse et J.-D. Weisz (200I), et une autre encore en sous la direction de Hugues Rabault (2016). Depuis deux décennies également de nombreux ouvrages en allemand et en anglais ont examiné à nouveaux frais l'ordolibéralisme allemand. Après avoir détaillé les apports du livre de Patricia Commun, nous présenterons quelques éléments en débats du point de vue de l'histoire de la pensée économique

\section{I.L'ordolibéralisme - une tentative d'approche synthétique}

Le livre est divisé en deux parties, la première est consacrée au «renouveau de la pensée libérale» allemande dans les années 1920-1940 tandis que la seconde traite de la «reconstruction d’un ordre libéral après 1945 ». La première partie ouvre la réflexion sur la genèse des idées ordolibérales par un chapitre retraçant l'évolution des traditions économiques allemandes dans les années qui suivent la Première Guerre mondiale. L'économie en Allemagne s'est développée comme une branche des sciences camérales enseignées à l'Université depuis l'époque moderne, de sorte que la quasi-totalité des économistes ont reçu une formation universitaire, quand ils ne sont pas eux-mêmes le plus souvent des universitaires, à la différence de la France où l'administration et les écoles d’ingénieurs occupent une place de choix dans la formation des économistes. À la fin du XIX $x^{e}$ siècle, les liens entre l'Empire wilhelminien et les économistes avaient été particulièrement étroits : les élites politiques et les grands noms de l'économie se retrouvaient non seulement dans le Social Verein mais aussi dans la Staatswissenschaftliche Gesellschaft fondée tout exprès à Berlin par Gustav von Schmoller, le leader de l'école historique. Aussi n'est-ce pas par hasard si la «loi économique» la plus célèbre attribuée à un économiste allemand du XIX siècle soit sans conteste la loi de Wagner qui 
stipule que la part des dépenses publiques est croissante du fait des pressions pour que le progrès social suive le progrès économique. C'est dire que la méfiance vis-à-vis des dépenses publiques que l'on trouve dans les traditions économiques françaises ou anglaises n'était alors pas de mise en Allemagne où le courant libéral (dit de Manchester) était marginalisé.

Le chapitre 2 est consacré à la période de l'après-guerre, marquée en Allemagne par l'hyperinflation des années 1922-1923, puis par la montée du chômage après la crise de 1929. Patricia Commun montre la participation des ordolibéraux aux débats contemporains. Ainsi Walter Eucken (1891-1950) voit, en 1923, dans l'endettement et le gonflement de la masse monétaire la cause de l'inflation. Wilhelm Röpke (1899-1966) dans Krise und Konjunktur (1932; 1936 pour la traduction anglaise, Crises and Cycles), avance une explication des crises qui anticipe celle que présentera Friedrich Hayek selon laquelle «c'est la création excessive de crédit par la Banque centrale qui fait baisser le taux d'intérêt en dessous du taux d'intérêt, d'équilibre» (p.44). Cet afflux de crédit permet alors des investissements importants dans l'industrie des biens d'équipement, et, a contrario, une épargne forcée dans les industries dont les profits sont inférieurs à l'inflation. Le surinvestissement est plutôt un «mal-investissement» (ibid.).

Röpke attribue l'origine de la crise à la mauvaise gestion de la monnaie et rejette la thèse de la crise inéluctable du capitalisme, comme celle de la sous-consommation. Quant aux remèdes pour limiter le chômage (réduction du temps de travail, interdiction du cumul, de l'immigration, et encouragement à l'émigration), ils sont vivement critiqués par Röpke, tout comme les propositions de dépenses publiques :

l'État ne devait en aucun cas se substituer aux investisseurs privés et dériver vers une politique économique dirigiste. Il était cependant la seule institution capable à la fois d'inspirer la confiance des créditeurs à l'étranger et de recréer la confiance nationale. Il fallait, pour ce faire, créer un élan initial qui serait à même de briser le pessimisme et le fatalisme ambiants que nourrissaient les haines anticapitalistes. Le rôle de l'État était plus celui d’un représentant de l'intérêt général, incitatif et psychologique, que celui d'un acteur économique (p. 58).

Dans le chapitre 3, Patricia Commun observe la réaction des libéraux allemands après l'arrivée des nazis au pouvoir le 30 janvier 1933. Elle examine en détail un article de Eucken, «Denken - warum?» (octobre 1933), dans lequel celui-ci défend la recherche d'une explication rationnelle du monde et rejette la «véritable hystérie collective antirationnelle et antilibérale» qui s'empare alors de l'Allemagne. Il soutient alors que les idées libérales ne conduisent pas au chaos et en appelle dans le cours de son raisonnement à l'ordre libéral. Dans la mesure où ce recours à l'ordre a été interprété par les adversaires de gauche des ordolibéralismes comme une collusion avec les nazis, Patricia Commun procède alors à la démonstration vigoureuse et convaincante que l'ordre dont il est question chez Eucken n’a rien à voir celui des nazis. Elle n’a pas de mal non plus à montrer l'opposition sans compromis au nazisme de Röpke et rappelle que pas moins de «252 économistes allemands vont devoir quitter leur pays» (p. 83). Ce faisant, elle retrace minutieusement la mise en place d'une résistance libérale dont la forteresse est 
l'université de Fribourg autour d'Eucken. Et, à juste titre, elle insiste sur la foi protestante qui anime la plupart des économistes ordolibéraux.

Le chapitre 4, est consacré aux Grundlagen der Nationalökonomie (Les Fondements de l'économie nationale, ouvrage d’Eucken paru en 1940). Après une analyse fouillée (50 pages), Patricia Commun conclut que «cette œuvre place Walter Eucken au cœur de la résistance libérale allemande. La notion d’ordre économique, de cohérence entre ordre politique et ordre économique, est devenue centrale dans toutes les discussions menées dans la clandestinité des organisations résistantes protestantes et conservatrices sur la nécessité d’un retour à une économie de marché après la guerre » (p. 153).

Le chapitre 5 , très court, est consacré à la rencontre avec le néolibéralisme français autour du colloque Lippman. Cette rencontre renforce l'intention des ordolibéraux allemands de mettre au cœur de leur programme la lutte contre les monopoles. La philosophie sociale d'un Louis Rougier va aussi influencer les ordolibéraux.

Le chapitre 6, qui clôt la première partie est consacré aux propositions d’Alexander Rüstow et Röpke. Après avoir défendu la logique du libéralisme, les deux auteurs vont critiquer la théorie économique libérale, surtout dans la présentation qu’en font les auteurs anglosaxons. Ainsi Rüstow reproche-t-il à la théorie économique libérale «sa cécité pour les problèmes de société et l'importance énorme des contraintes sociales hors de la sphère économique », tout en mettant en avant une opposition déterminée aux monopoles. Ce faisant, il cherche à définir un programme de troisième voie qui éviterait les écueils à la fois du collectivisme et du capitalisme en reposant sur une séparation stricte entre le pouvoir économique privé et l’État: «Ce dernier devait se garder d'interventions qui bouleversaient le marché en permanence et empêchaient une planification à long terme et une direction financière solide dans les entreprises. » (p. I70).

Pour sa part, durant ces années précédant la fin de la Seconde Guerre mondiale, Röpke prône une «économie humaine» fortement influencé par l'encyclique l'encyclique Quadragesimo anno proclamée par le pape Pie IX le I5 mai I93 I et rédigée par le jésuite allemand Oswald von Nell-Breuning, ainsi que le recueil d’articles Civitas Humana. Grundfragen der Gesellschafts- und Wirtschaftsreform, paru en Suisse en 1944 en témoigne.

Naturellement les classifications des thèmes économiques comprennent une division «systèmes économiques» dont le premier thème est «capitalisme». Mais, à juste raison, Patricia Commun parle, comme Röpke lui-même, d’un ordolibéralisme sociologique, puisqu'il s'agit là d’un discours sur les caractéristiques d’un système capitaliste idéal déconnecté des réalités économiques et notamment des conditions de faisabilité.

Tournée vers la «reconstruction d’un ordre libéral après 1945 », la seconde partie de l'ouvrage de Patricia Commun est davantage factuelle. Sans minimiser les dégâts occasionnés par les bombardements, elle rappelle que «la destruction du potentiel industriel est plus limitée que ne le suggère l'aspect désolant des villes bombardées. Grâce à un patrimoine 
industriel en augmentation constante depuis 1936, grâce à une activité industrielle tirée par la guerre, ainsi qu’à des bombardements aériens sélectifs de la part des alliés américains, le potentiel industriel fut relativement peu amoindri par la guerre » (p. 233, note 6). Mais les équipements sont une chose et l'organisation de la production en est une autre, en sorte que les limites d'une économie de commandement se font sentir dès 1947, au point que les puissances occidentales acceptent l'idée de «sauver» le maillon allemand de l'économie européenne. Encore faut-il convaincre la population que l'économie de marché est une meilleure option qu’une économie dirigée ou planifiée comme celles qui se mettent en place en France ou aux Pays-Bas. Le grand architecte de cette opération sera Ludwig Erhard (1897-1977) dont Patricia Commun connait particulièrement bien l'itinéraire qu'elle retrace avec brio dans le chapitre I de cette seconde partie soulignant en particulier l'utilisation de la coloration sociale pour «vendre »l'économie de marché.

Le chapitre 2 consacré à l'immédiat après-guerre traite de l'intervention dans la politique économique comme dans les débats sur les structures économiques des ordolibéraux. Ainsi le premier manifeste de l'ordolibéralisme, l’Ordojahrbuch, paraît en mai 1948, peu avant la mise en place de la réforme monétaire et de la libéralisation des prix - véritable «reset» de l'économie allemande. La revue s'ouvre sur un article de Hayek en défense du «vrai individualisme» qui donne le ton. Patricia Commun passe ensuite en revue les différentes nuances de l'ordolibéralisme. Elle oppose ainsi un «ordolibéralisme autrichien» représenté par Hayek pour lequel l'homme serait suffisamment «humble» pour respecter le cadre libéral à un «ordolibéralisme allemand» qui, instruit par la démiurgie nazie, insisterait davantage sur les différents moyens de limiter ces débordements. L'école de Fribourg a donc cherché du côté du droit les moyens de «faire obstacle à la croissance incontrôlée de tout pouvoir économique et politique» (p. 300).

Pour sa part, Röpke défendait les PME contre les grandes entreprises. La concentration, en renforçant le pouvoir de négociation des grandes entreprises, est défavorable aux travailleurs. Mais c'est surtout avec Alfred Müller-Armack (I90I-I978) que l'économie sociale de marché devient une composante importante de l'ordolibéralisme. Celui-ci, un temps séduit par le nazisme, se convertit à l'économie de marché à partir de 1943 à condition que l'ordre économique fasse sa place au «devoir de justice sociale». Il défend ainsi la création «d'un droit social, condition au bon fonctionnement de l'économie de marché » (p. 308).

Le Chapitre 3 montre comment les ordolibéraux, très réservés vis-à-vis de la cogestion et hostiles à des politiques un peu ambitieuses de redistribution, se sont fait les gardiens de l'ordre libéral dans l’Allemagne des années 1950 et 1960. Patricia Commun relève que l'indépendance des banques centrales, qui sera le grand totem libéral de la fin du $\mathrm{xx}^{\mathrm{e}}$ siècle, est absent des préoccupations ordolibérales dans les décennies de l'après-guerre. 
Le chapitre 4 rapporte comment Müller-Armack a réussi à infléchir selon les principes ordolibéraux la construction européenne dans les années I950. L’influence de la pensée des ordolibéraux s'étend à l'ensemble des pays européens via les analyses de l'OCDE. Patricia Commun conclut néanmoins sur l'inachèvement de l'ordre libéral en faisant état de l'écart entre la société et l'économie rêvées des ordolibéraux avec les compromis que constituent les institutions mises en place en Allemagne:

La grande déception des ordolibéraux est non seulement de voir les fruits de la prospérité trop rapidement dilapidés, mais aussi et surtout, en dépit de cette prospérité dont ils pensaient, à tort, qu'elle rendrait l'interventionnisme social de l'État moins indispensable, d'assister à la naissance et au développement d'un État-Providence qui sape les fondements économiques et humains de la société libérale et de l'individualisme libéral (p. 349).

Le livre sachève enfin par un chapitre consacré au dernier grand appel vibrant de Röpke en faveur d'un «ordre sociétal libéral en péril», Jensseits von Angelbot und Nachfrage (1958; 196I pour la traduction en français, Au-delà de l'offre et de la demande, vers une société humaine). Röpke s'y émeut des dangers apportés par la croissance économique - on commence à parler de la société de consommation vis-à-vis de laquelle la litanie des plaintes semble illimitée. Röpke est intarissable quand il s’agit de décrire les méfaits de la «croissance quantitative» mais il dénonce également le péril que représente selon lui la croissance de la population et son enchainement diabolique : «plus les naissances sont nombreuses et plus la demande est forte, plus les investissements se multiplient et plus le plein-emploi est réalisé, plus la prospérité est grande et plus le ressort de l'économie est vigoureux » (p. 353).

\section{Questions et perspectives}

Il faut savoir gré à Patricia Commun d’avoir réalisé cette synthèse d'auteurs prolifiques (la bibliographie compte une quarantaine d'entrées pour Böhm, Eucken, Erhard, MüllerArmack, Röpke et Rüstow - surtout des livres. On apprécie particulièrement qu’elle se soit efforcée de donner la parole à ces auteurs à la faveur d'un choix de citations judicieux qui n'alourdit pas le texte mais au contraire fait progresser la présentation. En effet, loin d'être seulement une œuvre de paléontologie où il s'agirait de suivre les bifurcations et les évolutions d'une espèce disparue, l'arbre du libéralisme dont Patricia Commun cherche les racines chez Kant est toujours vivant. Et on la sent même persuadée de pouvoir tirer quelque enseignement contemporain de ce rejeton du $\mathrm{Xx}^{\mathrm{e}}$ siècle apparu dans les circonstances dramatiques de l'après Première Guerre mondiale à la suite d'une hybridation avec la réforme protestante. Le livre met bien en valeur les différentes dimensions de l'ordolibéralisme, économique, juridique, politique, sociologique et relie adroitement les approches ordolibérales à celles de la seule prix Nobel d'économie, Elinor Ostrom. Cependant, hormis l'introduction qui, à juste titre, insiste sur les raisons que nous fournit l'actualité de s'intéresser à l'ordolibéralisme, la sélection des auteurs et des thèmes comme leur ordonnancement relèvent d'une lecture propre que nous voudrions à présent questionner. 


\section{I. Les racines philosophiques}

Patricia Commun insiste sur les racines kantiennes. Elle rappelle que «le célèbre texte de Kant Qu’est-ce que les Lumières? (1784) est cité dans l'article de 1948 comme une référence évidente par Walter Eucken, qui adhère sans réserves à ce manifeste d'émancipation intellectuelle vis-à-vis de toutes les formes d’autorité, religion, État, philosophie, science et même histoire ou actualité» (p. 297). En même temps, dans un épisode de l'histoire des ordolibéraux, ils se sont appelés «ricardiens » ce qui marquait leur volonté d'une approche déductive, mais aussi leur retour aux classiques. Deux dimensions mériteraient de retenir l'attention : la dimension éthique du marché d'une part et la place de l'histoire d'autre part (traitée par Eucken dans son article de 1938 sur le dépassement de l’historicisme).

\subsection{La cogestion}

Les racines idéologiques de la cogestion remontent en Allemagne aux débuts de la société industrielle moderne. Déjà, dans la période Vormärz (I8I5-mars I848), des économistes libéraux et même conservateurs sociaux s'étaient prononcés pour une représentation des travailleurs dans les organes de direction des entreprises. Elle s'opéra finalement durant la guerre de I9I4 où des représentations des travailleurs furent instituées puis pérennisées par la constitution de la République de Weimar. La cogestion connut un nouveau départ dans des secteurs particuliers en 1947 puis fut étendue à toutes les entreprises de plus de 500 salariés. Ce trait du capitalisme allemand n'est mentionné qu'en passant par Patricia Commun qui rappelle simplement que les ordolibéraux étaient loin d'être unanimes sur la question. Elle est contenue «en germe » dans l’analyse du travail que fait Röpke en 1932 présentée page 50. Elle ne réapparait qu'à la page 228 où une note indique que «Ludwig Erhard et les ordolibéraux ne sont par exemple guère favorables à la cogestion (Mitbestimmung) qui se met en place progressivement à partir de 1952 ». Page 28I, elle nous indique que Franz Böhm et Walter Eucken en font un exemple de la mauvaise gestion engendrée par l'interventionnisme étatique dans la République de Weimar précipitant sa fin. À la page 309, la cogestion figure comme un des moyens d'assurer un «cadre où la justice sociale ne devait jamais se réaliser aux dépens de la liberté. ». Mais plus loin, aux pages 32 I-322, il est rappelé que les ordolibéraux (et en particulier Böhm) n’y sont guère favorables dans la mesure où ils la considèrent comme «une concession purement politique faite aux travailleurs pour les détourner du modèle communiste et acheter leur soutien au système libéral ». On voit toute la confusion qu'il y a à assimiler «économie sociale de marché » et ordolibéralisme, c'est un apport du livre et une question à creuser. 


\subsection{La monnaie}

Une autre surprise que réserve la lecture du livre vient du traitement de la Banque centrale. Patricia Commun écrit: «Il faut noter que le grand absent parmi les thèmes traités est celui du statut de la Banque centrale et de ou de l'indépendance de la Banque centrale. Cette thématique est à tort considérée comme un sujet ordolibéral central, dans la mesure où aucun des écrits ordolibéraux, et en particulier dans leur organe central l'Ordojahrbuch, ne traite de ce sujet» (p. 323).

Cette affirmation est appuyée sur le jugement du «meilleur connaisseur français du sujet», Éric Dehay:

La question de l'indépendance des Banques centrales n’a réellement été traitée dans la littérature économique que depuis une vingtaine d'années; ce traitement suit en fait l'histoire même de l'indépendance, apparue pendant l'entre-deux-guerres puis assez peu appliquée [...] il est quelque peu abusif de considérer que la théorie ordolibérale traite de l'indépendance. En effet, il ne s'agit pas d'une question qu'ont évoquée les principaux auteurs et fondateurs de cette école (cité par Commun, p. 243).

On peut légitimement mettre en doute cet argument d'autorité et il suffit pour cela de se rapporter à l’article de Röpke de 1952 «Kernfragen der Wirtschaftsordnung» («Questions centrales de l'ordre économique ») dans lequel il insiste expressément sur l'importance de «l'indépendance de la Banque centrale qui doit être garantie vis-à-vis de tous ceux qui ont un intérêt à ce qu'il y ait de l'inflation (en particulier un intérêt politique)» (Röpke, I997 [1953], p. 50 - traduit par nous).

Certes on peut interpréter en sens contraire les préconisations auxquelles était parvenu Eucken après analyse de l'hyperinflation. Ce dernier voulait une «constitution monétaire automatique » qui corresponde à un «stabilisateur de la valeur de la monnaie» et plaidait pour «une règle contraignante de la politique monétaire » (Eucken, 1968 [1952]). Peut-être faut-il admettre ici que nous avons un nouvel exemple des dangers du raisonnement par école. On peut trouver en effet des formulations plus ambiguës chez Eucken et naturellement Ludwig Erhard n'aurait sans doute pas apprécié d'avoir face à lui une Banque centrale qui poursuive des objectifs propres. Il est clair qu'à l'aune des débats actuels une clarification sur ce point serait bienvenue.

\subsection{L'ordolibéralisme et l'écologie}

Le dernier chapitre soulève une interrogation particulièrement actuelle. Il y a en effet dans l'ordolibéralisme une dimension environnementale largement passée inaperçue mais que l'on redécouvre aujourd'hui que la planète entre dans l'anthropocène. C'est ainsi que les imprécations de Röpke vis-à-vis de l'accroissement de la population et la destruction de la planète ne sont pas sans rappeler les positions de Bertrand de Jouvenel, affirmant un souci de protection de la nature du point de vue d'un conservatisme libéral. Il en va de même 
chez Eucken dont les préoccupations vis-à-vis des conséquences environnementales du système économique était très avances sur son temps et peut-être aussi sur le nôtre :

Le système économique fonctionne très bien mais il ne prend pas en considération les répercussions que la réalisation des plans individuels exerce sur l'ensemble des données sociale. Un exemple est fourni par la destruction des forêts de l'Amérique du Nord qui conduit à la détérioration des sols et du climat et au développement des prairies. Ou bien considérons les effets nocifs pour la santé qui sont la conséquence de l'industrie chimique et la pollution de l'eau. (Eucken, 1968 [1952], cité par Peukert, 1998. p. 125).

\subsection{L'économie de marché}

Finalement pour l'économiste ou l'historien de la pensée économique le livre de Patricia Commun est l'occasion d'un réexamen complet de la pensée économique allemande durant les deux premiers tiers du $\mathrm{xx}^{\mathrm{e}}$ siècle ainsi que des restructurations consécutives survenues dans d’autres pays voisins - réexamen qui, comme le souligne Andreas Renner, reste largement en chantier (Renner, 2000 a et b) . La pensée libérale en économie avait connu une «révolution» dans le dernier tiers du XIX ${ }^{e}$ siècle avec la fameuse révolution marginaliste et le triomphe de l'école néoclassique comme devait l'appeler Charles Gide. La formalisation (sauf pour l'école autrichienne) a été la caractéristique la plus marquante des néoclassiques. Cette école qui tend à devenir dominante au tournant du $\mathrm{XIX}^{\mathrm{e}}$ et du $\mathrm{Xx}^{\mathrm{e}}$ siècle, en prenant le pas tant sur l'école historique allemande que sur l'école classique, a été ébranlée par la crise de 1929. Les néolibéraux dont les ordolibéraux comme Hayek, Allais ou l'école autrichienne représentent des réponses aux défis que la Grande Crise a posé aux économistes comme Arnaud Diemer l'a montré en plusieurs articles (pour un bilan, voir Diemer, 20I7).

Mais au-delà des problèmes posés par les crises à la croyance en un retour automatique à l'équilibre, le fonctionnement des marchés a posé de redoutables difficultés aux modèles néoclassiques dont la construction reposait sur l'hypothèse d'une concurrence «parfaite», c'est-à-dire sur des marchés constitués de petites unités. Or la fin du XIX siècle a été marquée par la croissance de la taille des entreprises et un fort mouvement de concentration particulièrement marqué aux États-Unis et en Allemagne. Les réactions furent différentes dans les deux pays. Aux États-Unis, le Sherman Anti-Trust Act du 2 juillet I89o fut la première tentative de limiter les comportements anticoncurrentiels des entreprises : il marque la naissance du droit de la concurrence moderne qui allait fournir la matière d'une économie et d'un droit de la concurrence. Aux États-Unis, les économistes ont tenté dès avant la Première Guerre mondiale de combler l'écart entre la firme atomistique postulée par les modèles d'économie pure et la réalité. C'est ainsi que John Bates Clark (1847-1938) s'intéressait dès le tournant du siècle au contrôle du pouvoir de monopole. Avec son fils, John Maurice (1884-1963), il mettait en garde contre la toutepuissance des trusts et cherchait à définir les conditions dans lesquelles la concurrence pourrait s'exercer et développer ses bienfaits pour la société (Clark et Clark, I9I2 [190I]). 
John Maurice Clark allait proposer l'idée de «concurrence praticable » en 1926 jetant les bases de l'économie industrielle. Dans cet ouvrage, il développait l'idée d'une gouvernance économique nationale, détaillant les facteurs institutionnels, économiques et légaux susceptibles d’encadrer le comportement des monopoles (Clark, 1926).

Le contexte allemand présente un contraste marqué avec la situation américaine alors même que la croissance de la taille des firmes a été une caractéristique de la croissance allemande comme celle des États-Unis. Alors que le Shermann Act était mis en œuvre dès I890, légalisait la carellisation en I897 parce qu'«elle ne violait pas le principe de la liberté contractuelle». Comme le précisent encore Helmut Nees et Friedrich Beuth, «ce n'est qu'en 1923 qu'une règlementation des ententes a été adoptée, qui avait pour but d'empêcher l'abus du pouvoir économique, mais elle resta pratiquement sans effet sur le développement des cartels et des conglomérats (Konzern). Dans la période nazie le cartel a même été pris en charge par l'État et a débouché sur l'économie de guerre» (Nees et Beuth, 1980, p. I7). C'est cet état du droit de la concurrence qui fit de l'Allemagne «le pays des cartels»-(Commun, p. 86, souligné par nous).

Dans son livre, Patricia Commun souligne d'ailleurs que «le thème du contrôle des monopoles et des cartels va devenir essentiel dans la pensée ordolibérale» (p. 23) à la faveur de la réactualisation la pensée libérale. Elle signale que Rüstow a joué un rôle dans l'adoption du décret sur le contrôle des ententes de I923 tandis que Böhm dénonçait fermement, dès 1928, dans une publication intitulée Le Problème du pouvoir privé. Une contribution à la question des monopoles la tolérance des tribunaux vis-à-vis des abus des ententes (voir Commun, p. 86). De même en pleine controverse sur la CECA, Erhard s'élevait fortement contre les ententes : «Essayer d'échapper par des cartels aux fluctuations du marché et empêcher par une fixation collective des prix l'équilibre du marché ne peut contribuer qu'à l'aggravation des troubles et des tensions, et conduire finalement à une économie planifiée. » (Erhard, I968 [1952]).

Cette hostilité vis-à-vis des ententes marque une forte originalité des ordolibéraux - l'Allemagne n'a-t-elle pas été jusqu'à la seconde moitié du $\mathrm{xx}^{\mathrm{e}}$ siècle le pays par excellence des ententes («das klassische Land der Kartellen») au point que l'on est en peine de savoir qui en a décerné le label: Böhm en 1948 dans ou plutôt Gottfried Haberler dans son livre sur le commerce international 25 ans plus tôt où il affirmait que «l’Allemagne était le pays des cartels et de l'interventionnisme [et qu'] elle donnait le ton dans les cartels internationaux auxquels elle participait» (Haberler et al., 1933, p. 245). Il est certain que pour les libéraux cette préoccupation venait de loin et Laurent Guilhéry - que Patricia Commun cite dans une longue note - a récemment attiré l'attention sur la thèse d'Eucken qui était précisément consacrée au droit et à l'économie des ententes. Dans ce travail, Eucken prenait le cas des ententes dans le secteur du transport maritime - secteur de référence des cartels (jusque dans les années 1990) - dont il montrait la nocivité et il en tirait des conclusions en faveur d'une intervention forte de l'état «(subvention aux nouveaux entrants), régu- 
lation, contrôle des cartels». Il y a déjà là «les prémisses de sa pensée future : actions contre les pouvoirs de marché, engagement sans faille de l'économiste pour observer et dénoncer les pouvoirs de marché» (Guihéry, 2013, p. 250).

Il n'est pas étonnant dès lors que l'ordolibéralisme soit relu aujourd'hui par référence au néo-institutionnalisme. Ce rapprochement est devenu manifeste avec l'obtention par Ronald Coase du Prix Nobel, en 199I, dont l'apport était à cette occasion légitimement reconnu. Il revenait alors à Renner, un auteur en marge du monde académique allemand, de rappeler aux tenants du néo-institutionnalisme que Coase n’avait pas inventé l'analyse économique du droit et que déjà «un groupe d'économistes et de juristes avaient, dans les années 1930 et 1940, fondé l'école de Freiburg, même si elle ne se paraît pas du label "Droit et économie" » (Renner, 200ob, p. I64 - traduit par nous). I5 ans plus tard, Richter (2015) souligne la proximité entre l’approche morphologique d'Eucken et les styles de raisonnement des néo-institutionnalistes comme Oliver Williamson. Celui-ci d'ailleurs avait rendu hommage à la tradition allemande dans laquelle il cite non seulement l'école historique allemande mais aussi Karl Marx avant de mettre en exergue les apports d'Eucken (voir le chapitre 9 intitulé Transaction Cost Economics and Organization Theory dans Williamson, 1996).

\subsection{La macroéconomie de l'ordolibéralisme, préfiguration du keynésianisme} Comme souvent l'histoire de la pensée comme l'histoire tout court a tendance à revisiter le passé pour répondre à des problèmes actuels. Ce sont les difficultés monétaires européennes qui servent à Patricia Commun d'introduction à sa présentation de l'ordolibéralisme. De même, ce sont les interrogations quamène le retour des «grandes» crises comme celle de 2008 après celle de 1929 qui ont conduit Bertram Schefold à relire Müller-Armack et à y trouver une préfiguration de thèmes keynésiens (Schefold, 20I7). Ces traits keynésiens avaient déjà été relevés par Harald Hagemann en 1999 dans le livre de Röpke consacré à la conjoncture en 1932 (Hagemann, 1999). Mais il est remarquable que, dès 1929, Müller-Armack renverse la doxa néoclassique et anticipe la révolution keynésienne en attribuant le rôle moteur attribué à l'investissement qui lui-même dépend des anticipations : «le progrès capitaliste actif ne se finance pas à partir du revenu épargné et soustrait à la consommation, il n'acquiert pas son pouvoir dynamique du passé mais des chances de gain futur» (Müller-Armack, 1929, p. 654, cité par Schefold, 20I7, p. 68). Mais ce n'est pas seulement le crédit qui peut être la source d'une expansion, un accroissement de la quantité de monnaie, par exemple à la suite d’une entrée d'or en provenance de l'étranger, peut aussi avoir cet effet, ce que Müller-Armack précise :

L'aspect caractéristique curieux de cette expansion économique consiste dans le fait qu'il engendre lui-même les conditions qui garantissent son succès. Ce pouvoir d’achat supplémentaire n'a pas son origine dans le passé, il est seulement basé sur l'anticipation des revenus futurs, et il est essentiel de se rendre compte que cette anticipation de revenus futurs est la base à partir de laquelle la réalisation de ces futurs revenus sera possible (ibid.). 


\section{Conclusion}

Ainsi, beaucoup reste à découvrir dans l'ordolibéralisme si l'on veut comprendre le rôle qu'il a joué dans le développement économique allemand et européen. Nous avons essayé de replacer le replacer l'ordolibéralisme par rapport à des courants qui lui sont proches à l'étranger en ce qui concerne l'encastrement de la concurrence économique dans la société sans déborder du cadre temporel retenu par Patricia Commun. Mais il est clair que les avatars de l'ordolibéralisme durant les cinquante dernières années devraient fournir des clés pour comprendre l'histoire de la construction européenne et le développement du droit de la concurrence en Allemagne et en Europe jusqu'aux récents problèmes de l'euro. Le chantier est vaste et soyons sûr que nous aurons encore besoin d'être guidé par quelqu'un d'aussi expérimenté que l'est, sur ce sujet, Patricia Commun.

\section{BIBLIOGRAPHIE}

ALCOUfFE A., POETTINGER M. et SCHEFOLD B., 2017, Business Cycles: a History, London, Routledge.

BILGER F., 1964, La Pensée économique libérale dans l'Allemagne contemporaine, Paris, Librairie générale de droit et de jurisprudence.

BÖHM F., 1948, «Das Reichsgericht und die Kartelle: eine wirtschaftsverfassungsrechtliche Kritik an dem Urteil des RG. vom 4. Febr. 1897, RGZ. 38/|55», ORDO: Jahrbuch für die Ordnung von Wirtschaft und Gesellschaft, vol. I., p. 197-213.

BROYER S., 2007, La Pensée théorique et politique de Walter Eucken à la lumière des écoles historiques allemandes, thèse, université Lumière Lyon-2 - Université Goethe de Francfort-sur-le-Main.

CLARK J. B. et Clark J.M., 1912 (1901), The Control of Trusts. An Argument in Favor of Curbing the Power of Monopoly by a Natural Method, New York, Macmillan: $<$ https://archive.org/details/controloftrustsa00claruoft>.

CLARK J.M., 1926, Social Control of Business, Chicago, University of Chicago Press.

DIEMER A., 2017, From Ideas Cycle to Economic Cycle: The Refoundation of Liberalism (1929 to 1985), in ALCOUFFE, POETTINGER et SCHEFOLD, 2017, p. 173-188.

ERHARD L., 1952, «Kollektivismus ist Schuld — nicht Schicksal, Zehn Thesen von Professor Erhard zur Kartellfrage», Die Zeit, 24 juillet.

EUCKEN W., 1914, Die Verbandsbildung in der Seeschiffahrt, Dissertation, Universität Bonn. -, 1938, «Die Überwindung des Historismus», Schmollers Jahrbuch,. 62, p. 191-214.

-, 1940, Grundlagen der Nationalökonomie.

-, 1968 (1952), Grundsätze der Wirtschaftspolitik, Tübingen, J.C.B. Mohr. 
FÈVRE R., 2015, «Du libéralisme historique à la crise sociale du $x x^{e}$ siècle: la lecture de Wilhelm Röpke», Revue économique, 66-5, p.901-931.

-, 2017a, «Le marché sans pouvoir : au cœur du discours ordolibéral», Revue d'économie politique, |27-I, p. ||9-|5|.

-, 2017b, «Walter Eucken et Wilhelm Röpke face à la "nouvelle" question sociale», Revue d'histoire de la pensée économique, I-3, p. 209-240.

GUIHÉRY L., 2013, «Émergence de la concurrence dans les chemins de fer en Allemagne et en France: quels enseignements tirer de la thèse (1914) de Walter Eucken sur l'émergence de la concurrence dans le transport maritime au tournant du siècle dernier?», Revue d'économie régionale et urbaine, p. 231-255.

HABERLER G. et al., 1933, Enzyklopädie der Rechts- und Staatswissenschaft. Theorie der weltwirtschaftlichen zusammenhänge sowie darstellung und analyse der aussenhandelspolitik, XLI, Berlin, Verlag von Julius Springer.

HAGEMANN H., 1999, «The Development of Business-Cycle Theory in the German language area 1900-1930», Storia del pensiero economico, 37-I, pp. 87-122.

KOSLOWSKI P., 1998, The Social Market Economy: Theory and Ethics of the Economic Order. Berlin, Springer-Verlag.

- (éd.), 2000, The Theory of Capitalism in the German Economic Tradition: Historism, Ordo-liberalism, Critical Theory, Solidarism, Berlin, Springer.

LABROUSSE A. et WEISZ J.-D., 200I, Institutional Economics in France and Germany: German Ordoliberalism Versus the French Regulation School, Berlin, Springer.

MONGOUACHON Cl., 2015, «Ordolibéralisme versus Néolibéralisme: antagonismes idéologiques et conséquences pratiques en droit européen de la concurrence », in S. Dormont et T. Perroud (dir.), Droit et marché, LGDJ, p. 21-44.

-, 2016, «Pour une concurrence régulée: l'apport de Leonhard Miksch», in RABAULT, 2016, p. $161-189$.

NEES H. et BEUTH F., 1980, Wettbewerbs- und Kartellrecht, Wiesbaden, Betriebswirtschaftlicher Verlag Gabler.

PEUKERT H., 1998, Walter Eucken (189I-1950) and the Historical School, in KOSLOWSKI, 1998, p.93-146.

PIES I. et LESCHKE M. (éds), 2000, Ronald Coase' Transaktionskosten-Ansatz, Tübingen, Mohr Siebeck.

RABAULT H. (dir.), 2016, L'Ordolibéralisme: aux origines de l'École de Fribourg-en-Brisgau, Paris, L'Harmattan.

RENNER A., 2000a, «Die zwei "Neoliberalismus"», Fragen der Freiheit, 256, p.48-64. 
-, 2000b, «Ökonomische Theorie des Rechts: Ronald H. Coase meets Walter Eucken», in PIES et LESCHKE, 2000, p. 164-172

RICHTER R., 2015, German «Ordnungstheorie» from the Perspective of the New Institutional Economics, in Id. Essays on New Institutional Economics, 2015, Cham [u.a.], Springer, p. 161-183.

RÖPKE W., 1932, Krise und Konjunktur, Leipzig, Quelle und Meyer.

-, 1961 (1958), Au-delà de l'offre et de la demande, vers une économie humaine: économie de marché et collectivisme; la société de masse moderne; conditions préalables et limites de marché, Paris-Lausanne, Payot.

-, 1997 (1953), «Kernfragen der Wirtschaftsordnung», ORDO: Jahrbuch für die Ordnung von Wirtschaft und Gesellschaft, vol. 48, Soziale Marktwirtschaft: Anspruch und Wirklichkeit seit fünfzig Jahren, p. 27-64.

SCHEFOLD B., 2017, «Kaldor and Robinson on the Business Cycle: A Closed Chapter of Post-Keynesian Economics?», in ALCOUFFE, POETTINGER et SCHEFOLD, 2017p.65.

SOLCHANY J., 2015, Wilhelm Röpke, l'autre Hayek: aux origines du néolibéralisme,. Paris, Publications de la Sorbonne.

WILLIAMSON O., 1996. The Mechanisms of Governance, New York, Oxford University Press. 